\title{
Dinámicas y relaciones entre los emprendimientos, la formación del capital humano y los sistemas de producción de cinco empresas MiPymes del sector alimenticio en Bogotá
}

Fecha de recepción: 19 de enero de 2011

Fecha de aceptación: 28 de junio de 2011

\begin{abstract}
Víctor José Rodríguez Restrepo
vrodriguezrestrepo@hotmail.com

Universidad de la Salle

Administrador de empresas de la Universidad Externado de Colombia; especialista en Gerencia de talento humano y en Pedagogía universitaria de la EAN. Profesor investigador de la Universidad de la Salle. Profesor del área de emprendimiento y creación de empresa del Politécnico Grancolombiano.
\end{abstract}

\section{Resumen}

Este documento presenta los resultados de una investigación sobre las dinámicas y relaciones entre emprendimientos en cinco MiPymes del sector alimenticio en la ciudad de Bogotá. La pregunta a resolver en este estudio es qué tanto se han dimensionado estas empresas en el tema de la formación del capital humano para poder ser cada vez más competitivas y productivas y llegar a formar una cadena o red para el país, de tal forma que puedan abordar los mercados nacionales en el corto plazo y pensar en incursionar en los mercados internacionales al largo plazo, sabiendo que las posibilidades de mejorar la tecnología en las empresas son casi nulas.

Todas ellas son empresas manufactureras, unas más artesanales que otras, con poca capacidad de innovación, comprometidas en actividades comerciales y productivas. Siendo MiPymes del subsector de alimentos en el renglón de la panadería, se presentan los resultados observados y analizados a través de esta investigación.

\section{Palabras clave}

MiPyme, competitividad, capacitación, educación, tecnologías de la información.

\begin{abstract}
This document presents the results of research on the dynamics and relationships between enterprises in five my SMEs of the food sector in the city of Bogotá DC. The question to be answered in this study is how much have these companies been dimensioned in the topic of human capital training? All this in order for them to become more competitive and productive to create a chain or network for the country, so that they can address the national markets in the short term and think of entering international markets in the long term by knowing that the possibilities of improving technology inside the companies are almost null.
\end{abstract}

\section{Keywords}

MySME, Competitiveness, Training, Education, Information Technologies. 


\section{Introducción}

El siguiente trabajo tiene como propósito identificar los determinantes de las dinámicas organizacionales e interrelaciones entre los emprendimientos del sector de alimentos, la formación de capital humano y los procesos productivos de cinco MiPymes encuestadas en la ciudad de Bogotá. Los datos se obtuvieron en el mes de octubre del 2009.

En el tema del emprendimiento, se pretende examinar los apoyos recibidos por los emprendedores, tanto en el aspecto familiar como en el campo institucional, y los tipos de asesoría y consejo recibidos por las personas que han estado cerca de ellos para alcanzar a consolidar la empresa como tal. Se estudian además, los medios que intervienen en su desarrollo: tecnologías requeridas, conocimientos, habilidades, recursos y obstáculos. De otro lado, en el tema del capital humano, se estudiaron los principales problemas relacionados con la gente que se vincula a trabajar con ellos; la remuneración, el nivel educativo, qué piensan los emprendedores de la capacitación de su personal, el tipo de experiencia requerido, el perfil del empresario y su actuación de acuerdo a sus conocimientos y visión del negocio, la rotación presentada en sus niveles de personal y el componente salud como un factor determinante en las relaciones laborales.

De otra parte, se examinan las cadenas y redes con proveedores como un aspecto fundamental en el desarrollo empresarial, ya que a mayor diversidad de mercados, surgen más oportunidades de ser aprovechadas por las cadenas de abastecimiento por parte de las empresas Pymes del país. Aquí se estudia la localización, alianzas con los clientes, acuerdos, obstáculos y tipos de asociatividad.
También se examina el nivel de estas empresas frente a la competitividad de acuerdo a su tamaño de producción, sus presiones competitivas, los principales problemas de la empresa, si han intentado exportar, la utilización de los acuerdos comerciales y, en general, la experiencia en el ámbito exportador.

Se pretende conocer cómo estas empresas están frente a temas como la innovación, la creatividad y certificación tecnológica, por cuanto las empresas desarrollan hoy en día sus operaciones dentro de un sistema económico que condiciona sus pautas generales de actuación. Ese es el caso de Colombia, en donde en muchos campos de la productividad no se está certificado internacionalmente y es muy difícil presentarse ante el fenómeno de la globalización como empresas competitivas. Además no se innova por no contar con la debida investigación empresarial, por la carencia de recursos y el grado de sostenibilidad de las familias que están detrás de los emprendedores que, siendo más con espíritu combativo que cualquier otra intención, luchan por sobrevivir, subsistir y mantenerse y están muy lejos todavía de evolucionar y transformarse modernamente para adecuarse al contexto de una realidad mundial.

\section{Planteamiento del problema}

Al tratar de conocer las dimensiones del emprendimiento en cinco MiPymes de la ciudad de Bogotá con base en los entrevistados del sector de alimentos, se perciben las siguientes situaciones. Para 
empezar, todos los emprendedores se encuentran en la etapa de emprendimiento: su actividad se clasifica en el campo artesanal por cuanto utilizan más mano de obra intensiva sin calificación que verdadera tecnología. Siendo ésta más de incorporación de conocimiento empírico de sus dueños que una verdadera tecnología incorporada de ambientes y aprendizajes en la innovación tecnológica con el objetivo de generar nuevos conocimientos (Castellanos, 2003), mucho menos mecanizada, automatizada ni electrónica.

En segundo lugar, no tienen cómo acceder al componente financiero dada la poca posibilidad de armar un proyecto técnico que sea sustentado ante un organismo financiero que le permita una obtención de recursos y su posible desarrollo hacia el futuro. Además, no trabajan ningún modelo de asociatividad como: distrito industrial, pools de compras, redes de servicios o grupos de exportación, ni pertenecen a un clúster y mucho menos a una cadena productiva que los integre hacia una política concertada de crecimiento (García, 2008).

No tienen cómo innovar sus productos en el mercado, no poseen conocimientos para emprender de una mejor forma, ni hacen parte de redes institucionales para difundir sus productos y su empresa. Su dimensión emprendedora está en su capacidad personal pues dedican tiempo a la posibilidad de transformar los productos para el mercado y a conocer cómo se hacen las cosas de la mejor manera. Esto lo comparten con la familia y sus amigos como elementos motivadores del desempeño.

El emprendimiento dependerá en parte de la personalidad que poseen y del apoyo en una percepción total de la realidad en la que viven. Así mismo, de su capacidad de analizar las relaciones que se dan a su alrededor y de tener una actitud orientada a resolver los problemas del día a día para solucionar los problemas de subsistencia como la dificultad de empleo y las necesidades latentes del ámbito social.

\section{Marco teórico de la investigación}

El colombiano promedio utiliza el pan como complemento esencial del desayuno y en el resto de las comidas no entra a competir como un serio adversario, es por eso que la mañana es el campo de batalla en el cual el pan se tiene que batir con alimentos tan famosos y prestigiosos como la arepa. Tan es asi esta situación, que las últimas cifras indican que el 94,2\% de los colombianos consume pan para el desayuno y cada colombiano consume 24 kilos de pan al año mientras que en paises como Chile se consumen $96 \mathrm{ki}$ los en promedio (http://www.revistabarra.com.co).

Tabla 1. Participación en el mercado de acuerdo a la agroindustria a la que pertenece.

\begin{tabular}{|l|r|}
\hline \multicolumn{1}{|c|}{ SUB-SECTOR ALIMENTOS } & $\begin{array}{c}\text { PARTICIPACIÓN } \\
\text { PORCENTUAL }\end{array}$ \\
\hline Carnes y pescados & $18 \%$ \\
\hline Frutas, hortalizas, aceites y grasas & $15 \%$ \\
\hline Productos lácteos & $20 \%$ \\
\hline Productos de molinería & $29 \%$ \\
\hline Productos de panadería & $8 \%$ \\
\hline Otros & $10 \%$ \\
\hline Total & $100 \%$ \\
\hline
\end{tabular}

Fuente: Dane, Bancoldex, muestra

manufacturera mayo de 2008. 
Como se puede apreciar, las empresas que se tomaron como muestra para esta investigación pertenecen a dos de los subsectores que presentan una participación conjunta del 31\% del gran sector de alimentos en el país. El subsector de la industria panificadora en Colombia muestra que cada habitante consume en promedio casi 24 kilos de pan al año. Esto, producto de las nuevas tendencias alimenticias de la población a consumir cada vez menos harinas y a remplazarlos por productos cada vez más saludables de origen vegetal.

Fedemol, la Federación de molineros del país, es considerado el gremio que vela por los intereses de los empresarios que trabajan con el trigo. Esta entidad espera traer este insumo sin aranceles de Estados Unidos por cerca del 65\% de la importación para abaratar el producto a nivel nacional, ya que casi no se consigue pan de $\$ 200$ en las tiendas y panaderías de los sitios donde se vende y consume pan. Colombia cuenta con 41 molinos ubicados de manera distinta en los departamentos del país, su distribución para la producción industrial se encuentra repartida de la siguiente manera: (http:www. eltiempo.com, 2011)

Tabla 2. Distribución de la producción industrial de harinas en Colombia.

\begin{tabular}{|l|r|}
\hline 1.Panadería y pastelería & $67 \%$ \\
\hline 2.Pastas & $15 \%$ \\
\hline 3.Galletería & $11 \%$ \\
\hline 4. Consumo Doméstico & $7 \%$ \\
\hline
\end{tabular}

Fuente: repository.urosario.edu.co

El 98\% del trigo que se consume a nivel nacional es importado; el presidente del gremio comenta que el $70 \%$ del trigo del país se vende en panaderías y el porcentaje restante va a parar a la industria de alimentos para los animales (concentrados).

De otra parte, los productos de panadería se encuentran repartidos de la siguiente manera en las dos ciudades capitales más importantes del país

Tabla 3. Distribución del mercado de productos de panadería en medellín.

\begin{tabular}{|l|r|}
\hline \multicolumn{2}{|c|}{$\begin{array}{c}\text { DISTRIBUCIÓN DEL MERCADO DE PRODUCTOS } \\
\text { DE PANADERÍA EN MEDELLÍN }\end{array}$} \\
\hline Galletas & $32 \%$ \\
\hline Pan de trigo & $22 \%$ \\
\hline Fideos y macarrones & $12 \%$ \\
\hline Bizcochos pasteles y dulces & $8 \%$ \\
\hline Ponqués y tortas & $12 \%$ \\
\hline Otros & $14 \%$ \\
\hline Total & $100 \%$ \\
\hline
\end{tabular}

Fuente: http//repository.urosario.edu.co

Tabla 4. Distribución del mercado de productos de panadería en Bogotá D.C.

\begin{tabular}{|l|r|}
\hline \multicolumn{2}{|c|}{$\begin{array}{c}\text { DISTRIBUCIÓN DEL MERCADO DE PRODUCTOS } \\
\text { DE PANADERÍA EN BOGOTÁ D.C. }\end{array}$} \\
\hline Galletas & $23 \%$ \\
\hline Pan de trigo & $34 \%$ \\
\hline Fideos y macarrones & $10 \%$ \\
\hline Bizcochos pasteles y dulces & $13 \%$ \\
\hline Ponqués y tortas & $15 \%$ \\
\hline Otros & $5 \%$ \\
\hline Total & $100 \%$ \\
\hline
\end{tabular}

Fuente: cálculos aproximados por el autor.

La Federación de molineros, dentro de su paso en la modernización de la cadena del trigo, desarrolla un programa de capacitación y mejoramiento del proceso administrativo tanto de la calidad del producto como de la capacitación integral de sus propietarios, los panaderos. Lo anterior se viene gestando por el temor de la llegada al país de empresas panificadoras 
multinacionales con excelente calidad e intensas campañas publicitarias que puedan afectar las ventas por una parte $y$, por la otra, el interés del gobierno en que los Estados Unidos le dé el visto bueno al TLC (Tratado de Libre Comercio para Colombia).

La Fundación Carvajal fue la invitada por Fedemol a planear el curso para pequeños empresarios: En la actualidad se encuentran 800 agentes en todo el pais recibiendo capacitación, esta actividad está siendo apoyada por el Ministerio de Desarrollo Económico y la Corporación para el desarrollo de la microempresa. Este gremio sigue interesado en brindar cursos integrales de duración de seis semanas con 16 horas adicionales de asesoría técnica y administrativa de la Pymes para modernización del negocio (www.eltiempo.com).

\section{Desarrollo de la investigación}

Este trabajo partió de un listado de empresa Pyme obtenido del registro de sociedades en Bogotá sobre la pequeña y mediana empresa. Se tomaron 18 empresas al azar de un listado de más de 800 con las cuales se realizó el contacto a través de la facultad de Administración de empresas de Universidad de la Salle. Se dividió la muestra en once organizaciones que pertenecen al sector manufacturero, en su gran mayoría entidades dedicadas a la confección, y cinco pertenecientes al sector alimenticio. El estudio presentado es tan sólo de las empresas del sector de alimentos. El siguiente cuadro muestra los nombres y ubicación de las empresas encuestadas resultado de esta investigación:

\begin{tabular}{|l|l|}
\hline \multicolumn{1}{|c|}{$\begin{array}{c}\text { NOMBRE DE LA } \\
\text { EMPRESA }\end{array}$} & \multicolumn{1}{c|}{\begin{tabular}{c}
\multicolumn{1}{c|}{ DIRECCIÓN Y } \\
ENTREVISTADo
\end{tabular}} \\
\hline $\begin{array}{l}\text { Laboratorios Yermain de } \\
\text { Colombia }\end{array}$ & Cra 8a No 4-29;Silvia Tacha \\
\hline Saboré & $\begin{array}{l}\text { Ave. 1a de Mayo No 68-73; } \\
\text { Giovanni Ávila }\end{array}$ \\
\hline Masa arepas Lilia & $\begin{array}{l}\text { Cra 31 No 41a-12 Sur; Wilson } \\
\text { Medina }\end{array}$ \\
\hline Productos El Rey & $\begin{array}{l}\text { No dieron información } \\
\text { completa }\end{array}$ \\
\hline Compañía de Levaduras \\
Leva pan & $\begin{array}{l}\text { No dieron información } \\
\text { completa }\end{array}$ \\
\hline Productos Tina & Calle 23a No 17-55 \\
\hline Obleada & $\begin{array}{l}\text { Cra 17 No 59-58; Ricardo } \\
\text { Mejía }\end{array}$ \\
\hline
\end{tabular}

Para efectos del trabajo de campo, los profesores responsables de esta investigación se hicieron cargo de dos entrevistas con los dueńos o administradores de cada uno de los negocios. El trabajo tuvo una duración de seis meses desde la planeación hasta la entrega de los informes. Se utilizó un cuestionario de preguntas cerradas con múltiples respuestas y la recolección de datos se hizo por fuentes primarias con observación, lo que llevó en el momento de la revisión, codificación y análisis de los resultados a considerar el estudio más de investigación cualitativa que cuantitativa por la forma como se lograron los datos e interpretaron los resultados.

\section{a. Emprendimiento}

Estas empresas fueron escogidas y estudiadas por arrojar muy poca información por la naturaleza de las agrupaciones. Estas están en la etapa inicial debido a que casi todas se encuentran en la fase de darle estructura a la MiPyme con el apoyo tanto de familiares en tres de ellas, como de amigos, para comenzar sus actividades. Con respecto al soporte financiero, éste es 
también precario porque ninguna tiene cómo avalar frente a la entidad prestadora de recursos financieros un proyecto o plan de negocio formal y escrito, técnicamente desarrollado que le permita presentarse con una idea clara ante la entidad competente que le indique para qué solicita estos dineros. Tan sólo dos de ellas lo han solicitado pero no a entidades financieras, sino a terceros con el consabido riesgo crediticio. Prefieren pagar altos intereses a terceros que adjuntar la documentación exigida. Estas empresas de alimentos se han podido mantener en el mercado producto de sus pocos ahorros en cuatro de ellas. Según la encuesta, ninguna de las siete ha recibido asesoría o investigación por parte de entidad alguna. La poca tecnología que desarrollan no alcanza al 10\% porque el otro $90 \%$ está representado en una mano de obra artesanal y no calificada. Los medios que utilizan para conocer sobre los avances de tecnología para su negocio son el Internet, las revistas especializadas y las alianzas con los proveedores. Ninguna ha podido presentar una forma distinta y proyectiva de desarrollo empresarial. Todas quieren crecer a mediano plazo, pero la pregunta es ¿podrán alcanzar este propósito en un medio tan competido y sin ninguna ayuda? Como obstáculos presentados para acometer la idea emprendedora señalan: el poco apoyo institucional en los campos administrativo, tecnológico y financiero, el desconocimiento de redes y cadenas productivas y su débil infraestructura a la hora de crecer. Tampoco se encuentran afiliados a un gremio, sociedad, asociación o cooperativa que los ayude e impulse en el tema de mejorar su empresarismo. También es de anotar que ninguno de los entrevistados está pensando en estudiar o mejorar sus conocimientos para visualizar y hacerle frente a la competitividad. Son personas que trabajan arduamente para sostenerse y que están llenas de aspiraciones, pero tardarán muchos años en alcanzar la meta anhelada. El individualismo y la desconfianza los caracteriza y, un imperante en la cultura empresarial MiPyme colombiana.

\section{b. Capital humano}

Es de destacar que estas cinco empresas cuentan con 28 empleados en planta según se visualiza en la gráfica siguiente:

Tabla 5. Número total de empleados en las empresas.

\begin{tabular}{|l|l|}
\hline EMPRESAS ENCUESTADAS & TOTAL EMPLEOS \\
\hline $\begin{array}{l}\text { 1. Laboratorios Yermain de } \\
\text { colombia }\end{array}$ & Diez personas \\
\hline 2. Saboré & $\begin{array}{l}\text { No suministró } \\
\text { información }\end{array}$ \\
\hline 2. Masa arepas Lilia & Diez personas \\
\hline 3. Productos Tina & Seis personas \\
\hline 4. Obleada & Dos personas \\
\hline
\end{tabular}

En laboratorios Yermain de Colombia, el 65\% corresponde a hombres y el 35\% a mujeres. En Masa arepas Lilia el 100\% corresponde a hombres. En productos Tina son seis personas en planta, todos hombres y en Obleada E U, hay un hombre y una mujer. Dentro de los criterios para elegir trabajadores en la vinculación se encontró la experiencia laboral como el más significativo, dado que en su mayoría son trabajos operativos o de supervisión. Le sigue en importancia las habilidades manuales, por cuanto esta aptitud es bastante relevante en las empresas de alimentos, en donde es considerada una competencia específica del saber hacer para su contratación. De igual forma, la atención al detalle 
y el sentido de la urgencia son consideradas habilidades fundamentales en este oficio. Los principales problemas abordados por estas empresas lo podemos presentar en la siguiente tabla:

Tabla 6. Problemas relevantes del talento humano.

\begin{tabular}{|l|l|}
\hline \multicolumn{1}{|c|}{$\begin{array}{c}\text { EMPRESAS } \\
\text { ESTUDIADAS }\end{array}$} & $\begin{array}{l}\text { PROBLEMAS RELEVANTES } \\
\text { DE TALENTO HUMANO }\end{array}$ \\
\hline $\begin{array}{l}\text { 1. Laboratorios Yermain } \\
\text { de Colombia }\end{array}$ & $\begin{array}{l}\text { Escaso compromiso del } \\
\text { personal; Difícil encontrar } \\
\text { gente con experiencia }\end{array}$ \\
\hline 2. Saboré & No dio información \\
\hline 3. Masa arepas Lilia & $\begin{array}{l}\text { Resistencia a la modernidad, } \\
\text { falta de personal } \\
\text { especializado y alta rotación } \\
\text { de personal. }\end{array}$ \\
\hline 4. Obleada & Escaso compromiso \\
\hline
\end{tabular}

Como se puede observar, la no calificación del personal es una barrera determinante en las empresas de mano de obra intensiva, en donde la urgencia por sacar producto al mercado para cubrir la demanda es lo tradicional y se pierde por parte de los directivos el compromiso con el personal operativo por el aprendizaje y adiestramiento del trabajador. Esto es inversamente proporcional al estudiar su grado de especialización con respecto al salario, que en muchos de los casos tan sólo alcanza al mínimo legal vigente. En la siguiente tabla podemos observar el ingreso promedio mensual de los trabajadores de producción de las empresas estudiadas.

Tabla 7. Ingresos promedio.

\begin{tabular}{|c|c|}
\hline EMPRESAS ESTUDIADAS & INGRESOS PROMEDIO \\
\hline 1. Obleada & $\$ 360.000$ vendedores \\
\hline 2. Laboratorios Yermain & $\$ 750.000$ operarios \\
\hline 3. Masa arepas Lilia & $\$ 624.000$ operarios \\
\hline 4. Productos Tina & $\$ 497.000$ operarios \\
\hline
\end{tabular}

ํำ
La vinculación del personal se realiza sin ningún tipo de rigurosidad técnica en el manejo del personal, dado que en la gran mayoría de estas empresas cuando se presenta la vacante, las recomendaciones de conocidos son las que hacen juego a la hora de llenarla. Es más, quien se va debe dejar una persona conocida a cargo. Para los cargos superiores llámese jefes o profesionales deben entregar hoja de vida a la gerencia para una entrevista personal, siendo estos vinculados en muy pocos casos. No hay un proceso formal establecido; se maneja de manera subjetiva o por recomendación. En muchos de los casos se llena la vacante sin un conocimiento claro de las competencias propias del cargo.

\section{c. Nivel educativo}

Al estudiar el nivel educativo alcanzado por los niveles de ocupación del personal de estas empresas encontramos lo siguiente:

Tabla 8. Nivel educativo alcanzado.

\begin{tabular}{|l|l|}
\hline EMPRESAS ESTUDIADAS & \multicolumn{1}{|c|}{$\begin{array}{c}\text { NIVEL EDUCATIVO } \\
\text { ALCANZADO }\end{array}$} \\
\hline 1. Obleada & $\begin{array}{l}\text { El propietario tiene nivel } \\
\text { medio y el personal, } \\
\text { básico. }\end{array}$ \\
\hline 2. Laboratorios Yermain & $\begin{array}{l}\text { Nivel profesional en los } \\
\text { directivos y medio en el } \\
\text { personal de planta. }\end{array}$ \\
\hline 3. Masa arepas Lilia & $\begin{array}{l}\text { El propietario y personal } \\
\text { de planta tienen nivel } \\
\text { medio. }\end{array}$ \\
\hline 4. Productos Tina & $\begin{array}{l}\text { El propietario tiene } \\
\text { nivel profesional y el } \\
\text { personal, básico. }\end{array}$ \\
\hline
\end{tabular}

Realmente los recursos humanos son poco calificados en este tipo de empresas. El desarrollo de las plantillas laborales 
aún sigue siendo considerado por los emprendedores como un gasto en el corto plazo, fácilmente renovable porque no existe por parte de los trabajadores adaptación, agrado ni sostenibilidad en sus puestos de trabajo. El nivel del personal medio entendido como operarios, supervisores y secretarias no pasa de la educación media y tampoco se ve la posibilidad de que a través de las empresas en donde prestan sus servicios exista preocupación por parte de sus propietarios o directivos por ver si este personal puede superarse, desarrollar o ascender. Toda la preparación dependerá del trabajador si es que desea destacarse, mas no para continuar allí, sino para tener oportunidades en empresas de mayor nivel organizativo. Los propietarios y algunos directivos no desean seguir estudiando; consideran que con los conocimientos propios de su profesión en el caso de ingenieros, administradores y gerentes, es suficiente y están más preocupados por tratar de sacar adelante el negocio para sobrevivir, que en estimar la real posibilidad de continuar en su preparación personal. De pronto no porque no lo deseen, sino porque las circunstancias del medio no lo permiten.

\section{d. Capacitación}

Ninguna de estas entidades del sector de alimentos se encuentra ofreciendo la capacitación al personal ni de manera privada ni a través del Sena. Un directivo comenta: Lo ideal es recibir la capacitación dentro de la empresa para no perder el tiempo en los desplazamientos de los trabajadores. Todos los encuestados la consideran necesaria porque los resultados los harían más productivos y competitivos y se podría plantear el trabajo en equipo que es la variable que ven con mayor deseo para que el trabajo sea más beneficioso para todos.

\begin{tabular}{|l|l|c|c|}
\hline \multicolumn{1}{|c|}{$\begin{array}{c}\text { EMPRESAS } \\
\text { ENCUESTADAS }\end{array}$} & \multicolumn{1}{|c|}{$\begin{array}{c}\text { VARIABLES } \\
\text { ESTUDIADAS }\end{array}$} & SI & NO \\
\hline $\begin{array}{l}\text { 1.Laboratorios } \\
\text { Yermain de } \\
\text { Colombia }\end{array}$ & $\begin{array}{l}\text { Aprendizaje; } \\
\text { adiestramiento; } \\
\text { entrenamiento; } \\
\text { formación. }\end{array}$ & $\mathrm{X}$ \\
\hline 2.Saboré & $\begin{array}{l}\text { Aprendizaje; } \\
\text { adiestramiento; } \\
\text { entrenamiento; } \\
\text { formación. }\end{array}$ & $X$ \\
\hline 3.Masa arepas Lilia & $\begin{array}{l}\text { Aprendizaje; } \\
\text { adiestramiento; } \\
\text { entrenamiento; } \\
\text { formación. }\end{array}$ & $\begin{array}{l}\text { Aprendizaje; } \\
\text { adiestramiento; } \\
\text { entrenamiento; } \\
\text { formación. }\end{array}$ & $\begin{array}{l}\text { Aprendizaje; } \\
\text { adiestramiento; } \\
\text { entrenamiento; } \\
\text { formación. }\end{array}$ \\
\hline 4.Productos Tina & $X$ \\
\hline 5.Obleada & & \\
\hline
\end{tabular}

Los directivos no están siendo responsables ni conscientes por la eficacia de los trabajadores, ellos deben reconocer que la necesidad de aprendizaje es algo de lo cual nadie está exento. Un entrenamiento es bueno cuando una instrucción especifca está pensada para el trabajador en particular, o para un grupo de ellos. Cuando el directivo considere la posibilidad de ofrecer instrucción, piense primero en quien o en quiénes serán sus alumnos (Nilson, 1991).

\section{e. Experiencia}

En este sector de los alimentos, lo que se persigue es que las personas que llegan ya traigan la experiencia en este tipo de actividad, dado que lo que interesa 
realmente es que la persona sea hábil para el trabajo y lo realice de manera rápida, obviamente sin desmejorar la calidad del producto que va a salir al mercado. Este es el comentario de varios de los directivos encuestados.

En cuanto al perfil del empresario se obtuvo la siguiente información de quienes la suministraron:

\begin{tabular}{|l|l|c|c|}
\hline EMPRESAS ENCUESTADAS & \multicolumn{1}{|c|}{$\begin{array}{c}\text { PREPARACIÓN DEL } \\
\text { EMPRESARIO }\end{array}$} & $\begin{array}{c}\text { AÑOS DE EXPERIENCIA } \\
\text { LABORAL }\end{array}$ & $\begin{array}{c}\text { PROMEDIO DE } \\
\text { EDAD }\end{array}$ \\
\hline 1.Laboratorios Yermain & Profesional & 30 & $40-55$ \\
\hline 2.Productos Tina & Educación media & 30 & $40-55$ \\
\hline 3.Obleada & Primaria & 40 & $40-55$ \\
\hline
\end{tabular}

En términos de la formación, el personal de ventas está entre media y baja, porque las personas vinculadas en estas empresas no pueden pagar mejores salarios, entonces también es personal que carece de ciertas habilidades propias del campo de las ventas: la formación de vendedores es tan importante que sin ella estos no funcionan bien. Ciertamente hay empresas que no forman a sus vendedores (sea el caso el de estas empresas estudiadas); y consecuentemente venden mal o se aprovechan de los vendedores anteriormente formados (castells, 2000). El enseñar a vender debe partir de quien dirige los destinos de la empresa y debe comenzar por comunicarse con el cliente, localizarlo y entablar con él un dialogo que le permita identificar sus necesidades de producto y/o servicio. El vendedor tiene que saber negociar, aclarar dudas, enfrentar dificultades, persuadir y allanar el camino del cliente hasta cerrar la venta. $\mathrm{Mu}-$ chas de estas cosas requieren de un entrenamiento y puesta en práctica a través de la enseñanza por quienes orientan la empresa.

Las necesidades de formación se pueden clasificar en cuatro etapas: la formación específica que es aquella que trata del producto y la empresa, la formación genérica que tiene que ver con estrategias, técnicas y habilidades, el reforzamiento de la autoimagen y auto motivación y finalmente el plan de ventas (el y qué, cómo; cuándo dónde; cuántos son los que vamos a hacer).

\section{f. Salud}

La cobertura está dada por las EPS respectivas. Todas estas empresas mantienen a sus trabajadores y ellos mismos (personal directivo), amparados por lo reglamentado por la ley. Tan sólo dos empresas, Productos Tina y Obleada, realizan jornadas de capacitación en salud ocupacional, entendida ésta como la condición física y psíquica que se da en el trabajador como resultado de los riesgos a que se expone, derivados de su modo de incorporación en el proceso de trabajo en la actividad determinada: La ocupación es el desempeño de una determinada profesión u oficio bajo ciertas condiciones del ambiente concretas, condiciones que incluyen tanto del medio físico de trabajo como las formas de organización y control del trabajo. Es la condición física y psíquica que se da en el trabajador; con ello se quiere expresar que la salud es la condición que se representa en el trabajador concebido como un ser integral, es decir, como resultado de los riesgos a que se expone (Cuaderno de 
salud ocupacional para trabajadores centroamericanos, 1978). Los trabajadores sí realizan descansos regulares para tomar la media mañana o las onces de la tarde. A algunos les dan dos días de descanso en el mes, como es el caso de Productos Tina, sobre todo cuando se acerca la temporada de colocar producto en gran cantidad en el mercado. Todos los encuestados señalan como aspecto más importante para el buen desempeño laboral, la educación formal.

\section{g. Cadenas y redes}

En este ítem, que es tal vez uno de los más importantes de la investigación, se toma como hallazgo que no existe cadena, definida ésta como la cantidad que el comprador está dispuesto a pagar, en otras palabras, un precio. Porter (1995) llama cadena de valor a la red de actividades de una empresa, porque la empresa pretende transformar insumos a bajo costo en productos con un precio superior a los costos de la empresa (Mitzberg, 1997). Ésta debe estar organizada y conformada en el país de origen propio de esta actividad, ya que para proveeduría de insumos sí cuentan con empresas extranjeras, pero para sacar los productos a mercados internacionales, no. Mucho menos pensar en los clúster como estrategia de asociatividad empresarial.

Para el caso de las empresas que suministraron información, la investigación arrojó lo siguiente:
Los proveedores se convierten en la carta de presentación de cualquier empresa, son la imagen institucional ante terceros, de ahí que las relaciones comerciales bien manejadas hagan de la MiPyme y de la Pyme, un eslabón para su posible crecimiento. Sus clientes también son empresas tipo medio, más concretamente pequeñas empresas de los tres sectores de la economía del país ubicadas en la ciudad de Bogotá. No cuentan con alianzas con los clientes, por consiguiente tampoco se encuentran formalizadas. Ninguna de estas empresas pertenece a gremio o asociación alguna del país.

Un clúster en el mundo industrial (o clúster industrial) es una concentración de empresas, instituciones y demás agentes, relacionados entre sí por un mercado o producto, en una zona geográfica relativamente definida, de modo que al conformar en sí misma un polo de conocimiento especializado, tiene ventajas competitivas particulares. Este concepto fue popularizado por el economista Michael Porter en el año 1990, en su libro The Competitive Advantage of Nations ("Ventaja Competitiva de las Naciones").

En un mundo globalizado como en el que vivimos, las condiciones del mercado se presentan como una oportunidad para todas las Pymes. Para el caso colombiano es la salida a muchos de los problemas de carencia de tecnología, altos costos en la manufacturación de los productos, las sobrecargas laborales, el manejo de las distancias con el transporte y

\begin{tabular}{|l|l|l|}
\hline EMPRESAS ENCUESTADAS & TIPOS DE PROVEEDORES UTILIZADOS & $\begin{array}{c}\text { OBSTÁCULOS PARA AUMENTAR } \\
\text { PROVEEDORES }\end{array}$ \\
\hline 1.Laboratorios Yermain & Nacionales y extranjeros & Falta de competitividad en precios \\
\hline 2.Productos Tina & Nacionales y extranjeros & Falta de competitividad en precios \\
\hline 3.Obleada & Nacionales y extranjeros & Relaciones inestables \\
\hline
\end{tabular}


la dificultad del mismo. El negocio está en colocar productos suntuosos en mercados especializados. El clúster puede ser una de las salidas, en parte, al desarrollo de ciertos renglones de productos que al encontrar una red de empresas con insumos especializados, maquinaria, entidades financieras, gobierno, empresas de servicios, etc., que en cierta forma atraídas por el modelo de asociatividad a través de un clúster la interfuncionalidad e interdisciplinariedad de quienes lo conforman, ayuden a tener mejor negociación y concentración del mercado hacia el cual deben llegar.

A continuación se presenta un cuadro que ilustra cómo se presenta la situación de los clúster en América Latina con sus fortalezas y debilidades propias de la competitividad de esta parte del planeta.

Al analizar más específicamente los clúster en América Latina, Altenburg y
Meyer-Stamer (1999) sugieren una tipología distinta que refleja la diferencia de realidades entre los clúster internacionalmente competitivos y los de subsistencia a nivel local (www.iberpymeonline.org).

La situación de las pequeñas empresas de alimentos de la ciudad de Bogotá es la de clúster de subsistencia por la casi nula recurrencia asociativa, dado que la poca interacción entre empresas no es más que el producto de amistades entre colegas propietarios con escasa especialización, débiles en innovación y carentes de capital para invertir en la empresa, producto de su misma subsistencia social.

Una vez más aparece la concepción neoliberal del ser humano cuando se dice que el empleado es el capital más preciado, pero para echarlo a la calle como nunca antes en las contradicciones de la productividad y del mundo globalizado (Aktouf, 2001, p. 273).

Tipología de Altenburg/Meyer-Stamer de los clústers en América Latina

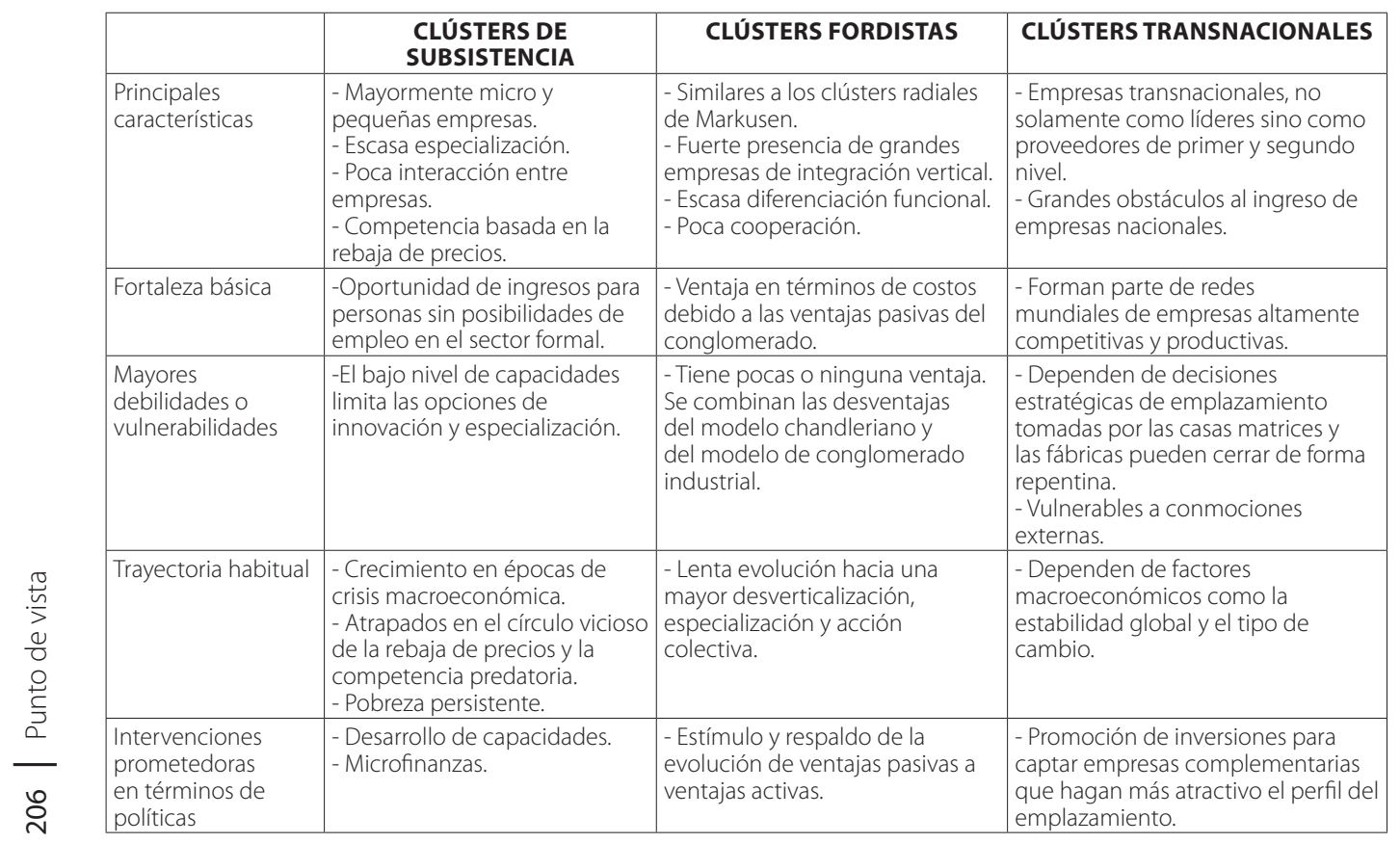




\section{h. Producción y competitividad}

Estas dos variables enfrentadas miden con propiedad el estado actual del sector de alimentos, estas MiPymes dejan ver a las claras su circunstancia presente. En el siguiente cuadro se puede apreciar productos que elaboran, si su nivel de competitividad ha mejorado o cambiado, la principal ventaja competitiva y las principales presiones que enfrentan.

\begin{tabular}{|l|l|l|l|}
\hline \multicolumn{1}{|c|}{$\begin{array}{c}\text { EMPRESAS } \\
\text { ENCUESTADAS }\end{array}$} & \multicolumn{1}{|c|}{$\begin{array}{c}\text { PRINCIPALES } \\
\text { PRODUCTOS }\end{array}$} & $\begin{array}{c}\text { PRINCIPALES VENTAJAS } \\
\text { COMPETITIVAS }\end{array}$ & $\begin{array}{c}\text { PRINCIPALES } \\
\text { PRESIONES } \\
\text { ENFRENTADAS }\end{array}$ \\
\hline 1. Laboratorios Yermain & Plantas, frutas y endulzantes & Alta calidad & Reducir tiempos y costos \\
\hline 2. Masa Arepas Lilia & Maíz, masa y arepas & Alta calidad & Reducir tiempos y costos \\
\hline 3. Productos Tina & Manjar blanco, bocadillo y brevas & Alta calidad & Falta de clientes \\
\hline 4. Obleada & Obleas decoradas & Alta calidad & Falta de créditos \\
\hline
\end{tabular}

Como se puede apreciar, en estas empresas están tratando de hacer las cosas lo mejor posible y encuentran problemas por los niveles de competencia tan grandes existentes en la ciudad. Sin embargo, todos manifiestan que han mejorado con el transcurrir del tiempo. Como principales problemas de la empresa se encuentran el cómo lograr más especialización en las tareas y tan sólo uno, el de Obleada, manifiesta competencia desleal. Ninguno de este sector realiza transacciones en el exterior ni está entre sus planes llegar a acuerdos comerciales porque son conscientes de la infraestructura y los dineros que requerirían para desarrollarse para una muestra exportable. A Laboratorios Yermain en épocas pasadas expo Pyme le colaboró para realizar algunos contactos con la gente de Venezuela y calificó esta ayuda como oportuna pero dice estar lejos todavía de convertirse en un potencial exportador.

Realmente aquí se confirma lo expresado por el Consejo privado de competitividad cuando dice: Somos poco productivos porque no poseemos mayor educación $y$ destrezas laborales.
Miremos el siguiente cuadro comparativo:

\begin{tabular}{|l|l|}
\hline Años de escolaridad adultos & $\begin{array}{l}\text { Colombia 7.9\%; } \\
\text { Estados Unidos 12\% }\end{array}$ \\
\hline $\begin{array}{l}\text { Tasa bruta de matriculados } \\
\text { en educación terciaria }\end{array}$ & $\begin{array}{l}\text { Colombia 28\%; } \\
\text { Estados Unidos 88\% }\end{array}$ \\
\hline $\begin{array}{l}\text { Calidad de educación en } \\
\text { ciencia y matemáticas }\end{array}$ & $\begin{array}{l}\text { Colombia 35\%; } \\
\text { Estados Unidos 56\% }\end{array}$ \\
\hline
\end{tabular}

Fuente: Consejo privado de competitividad-2007

(educación, pertinencia y destrezas laborales).

Un trabajador más capacitado y con mayor nivel educativo tiene mayor probabilidad de conseguir un empleo productivo en el sector formal (Informe de competitividad, 2007 Consejo privado de la competitividad).

\section{i. Innovación y certificación tecnológica}

En el presente cuadro se resumirá la situación de estas empresas del sector alimentos en materia de innovación y manejo tecnológico. 


\begin{tabular}{|l|c|c|c|}
\hline EMPRESAS ENCUESTADAS & $\begin{array}{c}\text { CERTIFICACIÓN DE } \\
\text { CALIDAD }\end{array}$ & $\begin{array}{c}\text { TECNOLOGÍA } \\
\text { INFORMÁTICA }\end{array}$ & $\begin{array}{c}\text { RAZONES DE IMPLANTAR } \\
\text { LA TECNOLOGÍA }\end{array}$ \\
\hline 1.Laboratorios Yermain & No & No & No \\
\hline 2.Masa Arepas Lilia & No & Sí & Agilizar los procesos \\
\hline 3.Productos Tina & No & No & \\
\hline 4.Saboré & No & No & No \\
\hline 6.Obleada & No & No & \\
\hline
\end{tabular}

Todas estas empresas desean tener sistematizados sus procesos, pero no cuentan con los recursos suficientes para hacerle frente a las aplicaciones de tecnologías de la información. Saben que con una página web podrían establecer un mejor contacto con el cliente, proveedores y llevar de mejor manera los aspectos contables. Por un lado, no todos los directivos se encuentran capacitados para hacerle frente al uso de las tecnologías y por el otro, se requiere de capital para poderlas implementar y no lo poseen. En términos generales no se alcanza un $10 \%$ en computadores personales por cada empresa para que de una u otra manera se cuente con procesos informáticos más depurados.

Las empresas de hoy deben realizar un gran esfuerzo para adaptarse a los cambios tecnológicos, sobre todo hoy en día cuando se habla de la cultura de la innovación empresarial; para propiciar la apertura de lo mejorable, innovar es sinónimo de emprender. Las sociedades más avanzadas y modernas son aquellas que son capaces de generar innovaciones en sus procesos empresariales. La cultura de la innovación se puede entender como una forma de pensar y de actuar que genera, desarrolla y establece valores, convicciones y actitudes propensos a satisfacer, asumir e impulsar ideas y cambios que suponen mejoras en el funcionamiento y eficiencia de la empresa, aún cuando ello implique una ruptura con lo convencional o tradicional (Patricio, 2007, p. 67).

\section{Consideraciones finales}

Una vez aplicada la encuesta y analizada la información recolectada se puede concluir lo siguiente. Estas empresas del sector alimentos MiPymes y Pymes de la ciudad de Bogotá, no cuentan con personal debidamente preparado y capacitado para enfrentar los retos que demanda la competitividad del momento. Estas organizaciones trabajan por su subsistencia y cuentan con bajos niveles de especialización e innovación en su cultura empresarial. Su principal esfuerzo lo realizan tratando de vincular personal con varios años de experiencia laboral compensando con sueldos bastante bajos; el fin es el de producir para los pequeños mercados que atienden. Se argumenta que buscar más clientes es una de las mayores dificultades, otro obstáculo está determinado por la reducción permanente de tiempos en los procesos productivos, al igual que la disminución de los costos para tratar de hacer más viables los precios de venta de sus productos, y así poder competir de mejor forma en los mercados nacionales.

No hacen uso del SENA como ente piloto de la capacitación de mandos operativos de las empresas del país. Sin 
embargo, pagan los parafiscales y están al día en materia legal. Los dueños o gerentes de empresa no se preocupan por mejorar los talentos de la empresa en materia de conocimientos y habilidades porque prefieren tener a sus trabajadores ocupados más que cualquier otra cosa. Los recursos son exiguos para desarrollar este programa. Sin embargo, el sector de transformación de estos insumos ha entrado en el camino de entregarle formación a sus afiliados a través de la Fundación Carvajal en asocio con el Gobierno Nacional, para matricular a los trabajadores en programas de preparación para la competitividad, por la presencia del paradigma del TLC como una incidencia de nivel macro para el país. De ahí que aparezca un factor interactuante: $a$ través de las condiciones propias del pais de origen de la producción y los destinatarios demandantes del producto (para este caso Colombia), la situación del pais determina el nivel de consumo interno del producto. La politica sectorial y de competitividad nacional que enmarca las condiciones de acceso y costo de recursos de capital, insumos para la producción (en este caso las harineras y las molineras); la cualificación de la mano de obra, las politicas y las reglamentaciones sobre contratación (para el caso nuestro MiPymes en su mayoría) y finalmente el orden monetario de las politicas cambiarias $y$ arancelarias influyen en la determinación de costos de producción y de capital (para la situación del sector, la poca infraestructura de las nuestras) (Espíndola, 2002, p. 6).

En el tema de la asociatividad a través de cadenas productivas o clúster, estas empresas no se intentan organizar en un sistema de alianzas, quizá por desconocimiento o quizá por carencia de visión empresarial y hasta de pronto individualismo cultural. Los perfiles de los empresarios que tienen a cargo la responsabilidad del personal, es el de personas mayores con buena pericia empresarial, en más de un caso, personas con más de 20 años de experiencia en labores propias de la industria y la comercialización de productos.

Impulsar el cambio no es tarea fácil, pero debe darse para estar a tono con los derroteros fijados por otras empresas en otros contextos del mundo globalizado. Mientras no se unan con un propósito común y acuerden una política clara de emprendimiento y superación personal en pro de sus organizaciones empresariales, estos valientes empresarios estarán expuestos al vaivén de los mercados y a perecer en el camino y sino a tardarse muchos años para llegar a ser grandes del empresarismo colombiano.

Se puede afirmar, después de analizar a los emprendedores entrevistados, que todos los acercamientos se dieron de manera empírica y que existen unos rasgos mayoritariamente psicológicos que caracterizan a estas personas, como la necesidad de logro e independencia para adquirir la libertad de sentirse dueños de sí mismos planteado por Mc Clelland (1972), su motivación, producto de la toma de riesgos para alcanzar el éxito a través de un conjunto de habilidades adquiridas, y las experiencias logradas en razón a su perseverancia, disciplina y organización del trabajo.

De otra parte, la conducta humana y los recursos también se convierten en factores determinantes del emprendimiento. Se puede percibir fácilmente a través de estos emprendedores pensadores, la búsqueda permanente de la creatividad por cuanto las oportunidades estarán determinadas por los recursos, pues, como lo planteó Stevenson (1990), 
el emprendedor es una persona con características especiales para determinar las ocasiones propicias para llevar a cabo la idea de iniciativa. Este comportamiento estará condicionado a la imposibilidad de predecir con exactitud los recursos necesarios para sacar adelante su proyecto de empresa.

Finalmente, las necesidades a escala humana que Neef (1995) plantea en su discurso ontológico, en donde se manifiesta que el individuo es producto del ser, el estar, el tener y el hacer como competencias de un emprendedor que lucha por la subsistencia en condiciones económicas que no son las mejores son también importantes, pues en últimas son el producto de las prácticas sociales y de los modelos políticos de quienes gobiernan que creen que están haciendo del desarrollo del país un gran programa, a costillas de las necesidades de la sociedad. No basta con darle a los bancos la posibilidad de liderar con préstamos a los pequeños empresarios, sino el establecer mecanismos de asociaciatividad, acompañamiento, formación específica y orientación en la aplicación de esos recursos.

Se recomienda entonces al sector $\mathrm{Mi}$ Pymes de alimentos lo siguiente:

La asociatividad empresarial como un buen mecanismo de cooperación entre empresas pequeńas y medianas, en donde cada empresa participante, manteniendo su independencia jurídica y autonomía gerencial, decide voluntariamente participar en un esfuerzo conjunto con los otros participantes para la búsqueda de un objetivo común.

Estos empresarios deben entender que el personal operativo debe capacitarse. Por consiguiente deben crear espacios durante la jornada laboral para asistir al SENA y recibir instrucción sobre BPM (Buenas prácticas de manufactura) y manipulación de alimentos, atención al cliente, etc., más aún cuando se pagan los parafiscales y se tiene derecho a la capacitación sin ningún costo, por parte del empresario - propietario.

El hacer uso de la creatividad y la originalidad apoyada en la experiencia de estas personas, permite generar nuevos productos que respondan a las necesidades del mercado, buscar reducir los costos, mejorar el servicio al cliente, detectar nuevos nichos de mercado y solucionar problemas de forma diferente a como normalmente lo realizan.

Mantener una excelente comunicación entre todos los pequeños a través de una red digital que les permita interactuar de manera permanente para anticiparse a los hechos y para establecer planes de contingencia futura que los oriente a actuar de manera oportuna y segura frente a los cambios del entorno.

Formular estrategias para sobreponerse a las insuficiencias del mercado de alimentos (transformación de la harina). Se trata de visualizar en materia de tecnología y de capital a través de la subcontratación con los grandes empresarios. Fomentar la investigación productiva $y$ las iniciativas de jóvenes productores con ideas innovadoras para lograr la mayor competitividad.

\section{Referencias}

I. Aktouf, O. (2001). La estrategia del avestruz racional (post globalización, economía y organizaciones). Facultad de Ciencias de la Administración de la Universidad del Valle. 
2. Angulo, MH. et al. (1993). El desarrollo de la personalidad emprendedora un programa de formación empresarial. Revista Escuela de Administración de Negocios, EAN. N 19.

3. Anzola Rojas, S. (1995). La actitud emprendedora. Espiritu que enfrenta los retos del futuro. México, Editorial Mc Graw Hill.

4. Alonso, C. (2009). De ejecutivo a empresario: reflexiones prácticas de un empleado convertido en empresario. Madrid: Editorial Díaz de Santos.

5. Andriani S, C. et al. (2003). Un nuevo sistema de gestión para lograr Pymes de clase mundial. México, Editorial Norma.

6. Beltrán, A., Torres, E. et al. (2004). Pymes un reto a la competitividad. Facultad de Administración de empresas Universidad Externado de Colombia., 1a Edición.

7. Benavides Espíndola, O. (2002). Competencias y competitividad (Diseño para organizaciones Latinoamericanas). Bogotá, Editorial Mc Graw Hill.

8. Castel Artal, M. (2000). Dirección de ventas (organización del departamento de ventas-gestión del vendedor). Madrid: Editorial ESIC (Escuela superior de gestión comercial).

9. Cardona, M. et al. (2008). "Las Dimensiones del emprendimiento empresarial". Experiencia de los programas cultura y fondo Emprender en Medellín.

Io. Consejo Superior Universitario Centroamericano de Salud Ocupacional para trabajadores..1966, ONU - CEPAL. Tegucigalpa, Honduras

I I.Castillo, A. (1999). Estado del arte en la enseñanza del emprendimiento. En Emprendedores como creadores de riqueza y desarrollo regional. INTEC-Chile. P. 1-21.
I 2.Cardozo, PP. et al. (2007). Las Pymes bogotanas y sus procesos de internacionalización. Un primer balance. Revista Panorama, $\mathrm{N}^{\circ}$ 3. Politécnico Grancolombiano.

I 3. Cámara de comercio de Bogotá. (2006). Asociatividad empresarial (estrategia para la internacionalización Memorias Consejo Regional de Competitividad de Bogotá y Cundinamarca.

I 4. Dávila Ladrón de Guevara, C. (1985). Teorias organizacionales (enfoque critico). Bogotá, Editorial Mc Graw Hill.

I 5.Drucker, P. (1964). Managing for Results. Nueva York: Harper \& Row.

I 6. (1986). The Frontiers of Management. Inglaterra: First plume Printer.

I7._-____________ (2005). Innovation and Entrepreneurship; practice and principles.

I 8. Freire, A. (2005). Pasión por emprender (De la idea a la cruda realidad). Bogotá, Editorial Norma.

I9. Filion, L. (2002). Emprendedores y pequeños propietarios de la pequeña-mediana empresa, PYME. Revista Administración y Organizaciones. Julio de 2003. p. 113-152.

20.Holland, J. (2001). Corporate value creation. Department of Accounting \& Finance University of Glasgow Working Paper Series.

21.Lemaitre, P. y Maders, HP. (1991). Cómo mejorar la organización Administrativa. Barcelona: Ediciones CEAC.

22.Lerma Kirchner, A. et al. (2008). Liderazgo emprendedor (Cómo ser un Emprendedor de éxito y no morir en el intento). España: Cengage Learnig.

23. Morcillo, P. (2007). Cultura e innovación empresarial. España: Cengage Learnig. 
24. Motta, PR. (2001). Transformación organizacional. Bogotá: Ediciones Uniandes, Editorial Alfaomega.

25. Nilson, C. (1994). Cómo formar a su personal. España: Ediciones Granica.

26. Neef, M. (1998).Desarrollo a escala humana. Barcelona (España).Editorial Nordan Comunidad.

27. Pallares Villegas, Z. (2003). Asociatividad Empresarial (estrategia para la competitividad).Bogotá D.C.; Colombia, Fondo editorial Nueva Empresa.

28. Porter, M. (1995). Ventaja competitiva de las naciones (estrategia competitiva). México: Compañía Editorial Continental.

29. Pinto Saavedra, JA. (2001). "Decálogo para la recuperación de la Economía productiva"; CINSET.

30.Pallares Villegas, Z. (2003). Asociatividad empresarial (estrategia para la competitividad). Fondo editorial Nueva Empresa.

31.Quintero U., VM. (1991). Alternativa micro empresarial. Facultad de Economía Universidad de San Buenaventura de Cali (Colombia). P. 289-327.
32. Rogers, C. (1951). El Proceso de convertirse en persona. Buenos Aires: Editorial Paidós.

33. Stevenson, H.H. y Jarillo, J.C. (1990). A Paradigm of Entrepreneurship: Entrepreneurial Management. Strategic Management Journal. 11: 17-27.

34. Suárez Ramírez, LF. (2005). Desarrollo del espíritu emprendedor. Universidad Nacional Abierta y a Distancia.

35.Soto Pineda, E. y Dolan, S. (2003). Las Pymes (ante el reto del siglo XXI, los nuevos mercados globales). México, Editorial Thompson.

36.Serna Gómez, H. y Suárez, E. (2005). La empresa familiar (Estrategias y herramientas para su sostenibilidad y crecimiento). Bogotá: Editorial Temis.

37. Tarapuez Chamorro, E. y Lima Rivera, C. (2008). Creatividad empresarial. Ediciones ECOE.

38. Van den Berghe, R. (1999). Cómo crear y gerenciar su propia empresa. Bogotá, Editorial Universidad Nacional de Colombia. 